\title{
Weaving of Fabric for Meter-Scale Floor Touch Sensors Using Automatic Looming Machine
}

\author{
Seiichi Takamatsu*, Takahiro Yamashita, Tetsuhiko Murakami ${ }^{1}$, \\ Atsushi Masuda ${ }^{1}$ and Toshihiro Itoh \\ National Institute of Advanced Industrial Science and Technology, \\ 1-2-1, Namiki, Tsukuba, Ibaraki 305-8564, Japan \\ 'Industrial Technology Center of Fukui Prefecture, \\ 10, Kitainadam 61 Kawai-washizukamatchi, Fukui, Fukui 910-0102, Japan
}

(Received January 22, 2014; accepted July 16, 2014)

Key words: weaving, automatic looming machine, touch sensor, fabric, conductive polymer

We have proposed and developed a 1.2-m-wide automatic looming machine to continuously construct a meter-scale floor touch sensor fabric. The fabric floor touch sensors are woven fabric with conductive-polymer-coated sensor fibers, and projection capacitive measurement is utilized for a human touch sensing mechanism. The automatic looming machine for floor touch sensors can weave sensitive sensor fibers as wefts and warps of the sensor fabric. For weaving different tensions for sensor fiber warps, a specific warp beam of sensor fibers is set behind the weaving machine to control the tension of the sensor warps. On the other hand, new linear actuators for the weft sensor fiber are added to weave the sensitive weft without destructive friction between sensor wefts and warps. Weaving of a $1.2 \times 5 \mathrm{~m}^{2}$ fabric with a $5 \mathrm{~cm}$ pitch is demonstrated using the developed looming machine. The pitch of the sensor fibers is changed from $5 \mathrm{~mm}$ to $1 \mathrm{~m}$. The capacitive sensitivity to touch input is tuned by controlling the coverage of sensor fibers with dielectric conventional polyester fibers. The output capacitance change at the smallest coverage of $25 \%$ is approximately twofold that at the coverages of 75 and $100 \%$. These weaving sensor fabrication techniques will provide low-cost and large-area sensor systems of floor touch sensors in nursing homes and hospitals.

\section{Introduction}

A meter-scale large automatic looming machine has been developed and used in a weaving fabrication process for a meter-scale floor touch sensor fabric for constructing human position or walk sensors because human height is in meter scale (i.e., the average height is $1.7 \mathrm{~m}$ ) and the area of rooms in homes is several square meters. ${ }^{(1-4)} \mathrm{In}$ particular, for human position sensing, a 1.2-m-wide looming machine was constructed

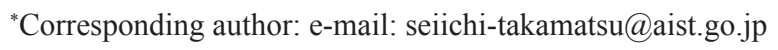


to weave a sensitive sensor fiber coated with a conductive polymer, and the forming of a 1.2 $\times 5 \mathrm{~m}^{2}$ sensor fabric with a $5 \mathrm{~cm}$ pitch of sensor fibers (i.e., average foot heel width of 6.3 $\mathrm{cm})^{(5)}$ was demonstrated.

The demand for elderly care sensors such as meter-scale floor touch sensors has been rapidly growing in order to reduce the heavy burden of caregivers and improve the quality of life of the elderly population, which is increasing rapidly in Japan and other advanced countries. ${ }^{(6-8)}$ To meet these demands, several sensors have been constructed, such as cameras, ${ }^{(7)}$ infrared light sensors, ${ }^{(6,9)}$ and floor touch sensors. ${ }^{(2,6)}$ Among these sensors, floor touch sensors have attracted the most attention. Floor sensors can monitor elderly people while maintaining their privacy because the floor sensor detects only the touch contact instead of images of a person. ${ }^{(7)}$ In addition, floor touch sensors can detect people in large areas of entire rooms, while infrared sensors are expensive, and dead areas of the sensors are unavoidable because of the small number of infrared sensors that are placed in a room. ${ }^{(6,10)}$ To monitor the elderly in the rooms, the sensor should be meterscale, because the beds are $1 \mathrm{~m}$ wide and $2 \mathrm{~m}$ long and the floor is 2 or $3 \mathrm{~m}^{2}{ }^{(5,6)}$ However, meter-scale touch sensors are difficult to fabricate using the current sensor manufacturing technology. ${ }^{(11-13)}$

The critical problem of meter-scale floor touch sensors is their size: the sensors are not several millimeters squared ${ }^{(11-13)}$ but several meters squared. The existing fabrication technology of silicon-based micromachining provides 5-mm-wide small pressure or touch sensors with high accuracy, but they must be at least $30 \mathrm{~cm}^{(12,13)}$ owing to the silicon wafer size and the chamber size of machining tools. Because the control of high precision patterning or alignment and uniformity of thin-film deposition are required, micromachining is easy on a small area of the device wafer but not on a large one. In addition, precise machining of the whole $30 \mathrm{~cm}(8 \mathrm{inch})$ wafer is very expensive (i.e., tens of thousands of US dollars) because processing tools and materials are expensive. Thus, tens of thousands of sensors are constructed at one time during the fabrication process and then cut into tens of thousands of pieces for achieving very low cost sensor chips. ${ }^{(12,13)}$ The existing silicon microsensors are not suited for large-area devices. In the same way, manufacturing technology of a printed circuit board cannot provide meterscale pressure or touch sensors because the sizes are limited to several tens of centimeters and a large-area board is expensive. ${ }^{(14)}$

Scalable fabrication technology for floor touch sensors (i.e., e-textile),$^{(15-17)}$ which is based on textile technology, has recently attracted much attention because automatic looming machines are considered a highly mature technology in use since the industrial revolution, which can produce several meter-wide fabrics continuously at a low cost. ${ }^{(15-22)}$ However, with the present technology, the sensor fibers have been woven manually, and there are few examples of automatic weaving of sensor fibers because the touch sensor fibers are sensitive to the destructive tension and friction produced by automatic looming machines. ${ }^{(18-22)}$ In fact, wrong tension is applied especially in the case of warp sensor fibers, resulting in threads being cut when the wefts are interlaced since the sensor fibers have a larger diameter than those of other conventional polyester or other fibers. In addition, large destructive friction and its resultant tens of kilovolts of static electric charge break the conductive film on touch sensing fibers when weft sensor fibers are 
interlaced between warps. Therefore, previous studies ${ }^{(18-22)}$ utilized manual weaving machines. Professor Sato's group at Nagoya University has conducted important studies on fabric touch sensors for various applications including artificial robotic skin, wearable keyboards, and bed sensors. ${ }^{(18-20)}$ Several types of microstructures on fibers are constructed for shear force or other force sensing. These studies have led to highly functional sensors, but the sensor fabric is limited to tens of centimeters through hand weaving because the sensing electrode fibers are fabricated by thin-film deposition with spatter and dielectric film deposition of Parylene in a chemical vapor deposition chamber. $^{(20)}$ In other previous studies, our group $^{(21,22)}$ developed a scalable fabrication process for sensing fibers by utilizing a conductive polymer and a die-coating technique. The electrode films are coated on the fibers continuously. However, the sensor size was limited to a $15 \mathrm{~cm}$ width because the weaving of the sensors was performed by a manual weaving technique. ${ }^{(21)}$ Hence, an automatic looming technique has not yet been developed for a large-area floor touch sensor fabric.

To meet the requirement of automatic weaving for the floor touch sensor, we proposed and developed a 1.2-m-wide touch sensor looming machine that weaves sensing electrode fibers as wefts and warps [Fig. 1(a)]. Conductive-polymer-coated fibers are woven in horizontal and vertical axis directions to form a floor touch sensor fabric, and the projection capacitive measurement is utilized for a human touch sensing mechanism. The developed automatic looming machine has unique features for weaving sensing electrode fibers as shown in Fig. 1(b). For weaving different tensions for sensor fiber warps, the specific warp beam of sensor fibers is set behind the weaving machine to control the tension of the sensor warps [Fig. 1(b)]. This is because different diameters of fibers have different tensions, and if they are rolled in the same beams, one of the fibers is loosened and the fibers are cut by the weaving of the wefts. On the other hand, new linear actuators for the weft sensor fiber are added in order to weave a sensitive weft without destructive friction between sensor wefts and warps. As shown in Fig. 1(b), the

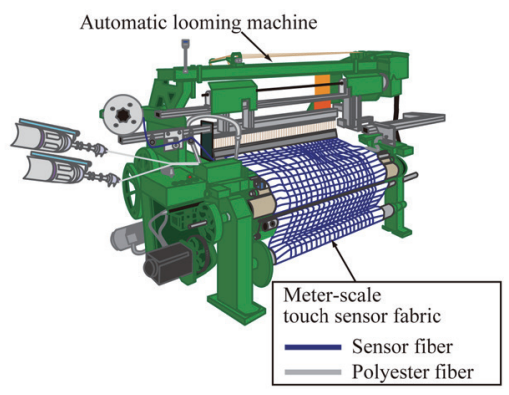

(a)

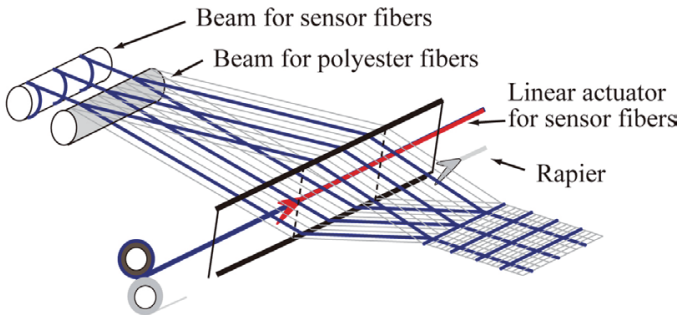

(b)

Fig. 1. (Color online) (a) Configuration of a developed automatic looming machine for floor touch sensor fabric. (b) Schematic view of automatic looming machines. The mechanism of our looming machine is different from that of the conventional ones consisting of a beam and a linear actuator. 
linear actuator takes sensor wefts and weaves them in the middle of the gap between open wefts while most repairs of the looming machines take and weave wefts just on the warps, and destructive friction between wefts and warps is generated. A $1.2 \times 5$ $\mathrm{m}^{2}$ fabric with a 5-cm-pitch sensor fiber is demonstrated to be woven by the developed looming machine, and its spatial resolution and capacitive sensitivity are controlled by the weaving technique.

\section{Fabrication Process and Structure of Meter-Scale Floor Touch Sensor Fabric}

Figure 2(a) shows the floor sensor structure, which is the woven fabric of conductivepolymer-coated sensor fibers as a weft and warps. The pitch of the sensor fiber is $5 \mathrm{~cm}$, and the sensor fibers work as an electrode to detect human feet. ${ }^{(5)}$ The touch sensing mechanism is based on the capacitance measurement between the sensing fiber and human body parts such as hands and feet, because the human body is conductive and works as an electrode. (22) In detail, the human body works as an electrode which is connected to the ground because the human body is large enough to work as a ground. The capacitance between the conductive polymer-coated fiber and human finger is measured as shown in Fig. 2(b). When the human finger touches the sensor fibers, a capacitor is formed between sensor fibers and the human finger. The transient current flows into the formed capacitor according to the capacitance of the capacitor if a pulsed voltage is applied to the sensor fibers. By applying a voltage to the sensor fiber and measuring the current, any capacitance increase under human touch can be detected. By detecting these capacitance increases caused by contact between the human body and sensing electrode fibers, the human's position is determined.

The meter-scale touch sensor fabric is continuously woven using our developed automatic looming system after hundreds of meter-long fibers are coated with a conductive polymer and dielectric polymers. The electrode sensor fiber structure that is used is a $1-\mu \mathrm{m}$-thick conductive polymer of poly(3,4-ethylenedioxythiophene):poly(sty renesulfonate) (PEDOT:PSS) (H. C. Stark, Clevios PH1000) and a 5-m-thick ultraviolet (UV)-curable polymer (Tesk corp. A-1864) on a 485- $\mu$ m-diameter Nylon fiber as shown

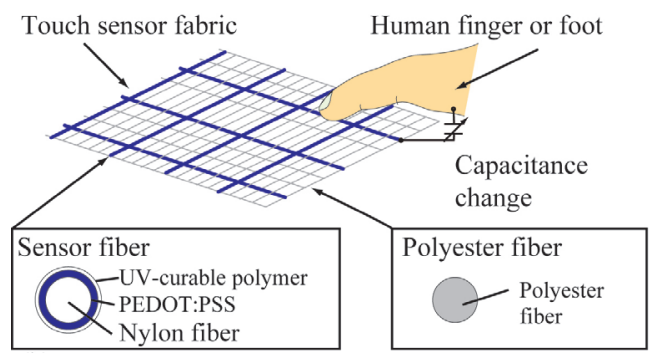

(a)

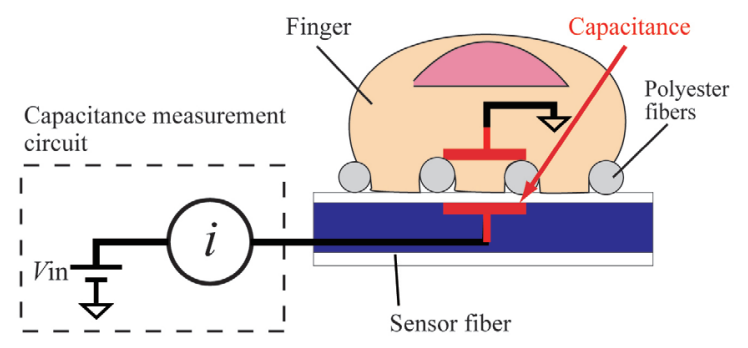

(b)

Fig. 2. (Color online) Structure of floor touch sensor fabric and human touch sensing mechanism. 
in Fig. 2(a). The coating is operated with a die-coating system that has a die for tuning the gap between fibers and dies and that coats the conductive polymer-water dispersion with the thickness of the gap between a fiber and the dies as described in the previous reports. ${ }^{(21,22)}$

In the developed automatic looming system, a conventional automatic looming machine was modified to weave the thick and sensitive sensing fibers as wefts and warps at the desired pitches. Figure 1(b) shows the specific features of an automatic looming machine for touch sensors, which has a new specific beam to wind out sensor fibers under certain tension. The looming machine has a new rapier of a linear actuator for sensing wefts that carries the wefts in the middle of the gap between the upper and lower warps in order to avoid any friction between warps and sensing fibers. Most rapiers carry the wefts only on the warp, resulting in destructive friction. Figure 3(a) shows the overall weaving system. Figure $3(\mathrm{~b})$ shows that the conventional polyester fiber bobbin and the sensor fiber bobbin are located on the left side of the automatic looming machines. Figure 3(c) shows the back side of beams of polyester fibers and sensor fibers. Two separated beams are equipped in the automatic looming system. The sensor fibers are wound out with certain tensions at the beam, and the fabrics are woven and rolled up at the cloth take-up roll. The polyester fiber and sensor fibers have different tensions owing to their different diameters and fiber numbers. Thus, the two beams have motors and control the tensions to the fibers. The beams can apply force ranging from 0 to $1.96 \mathrm{kN}$ to all warps. When only one beam instead of two beams leads to wrong tension to fibers, the space between the upper and lower warps narrows and the rapier cuts the warps at the time of carrying the wefts. The separate tension control of two different fibers with two beams will lead to the prevention of cutting of warps. On the other hand, wefts are interlaced by two rapiers [Figs. 3(c) and 3(d)]. The linear actuator rapier carries wefts only in the middle of the space between upper and lower warps, while the conventional rapiers carry wefts only on the lower warps, resulting in the destructive frictions.

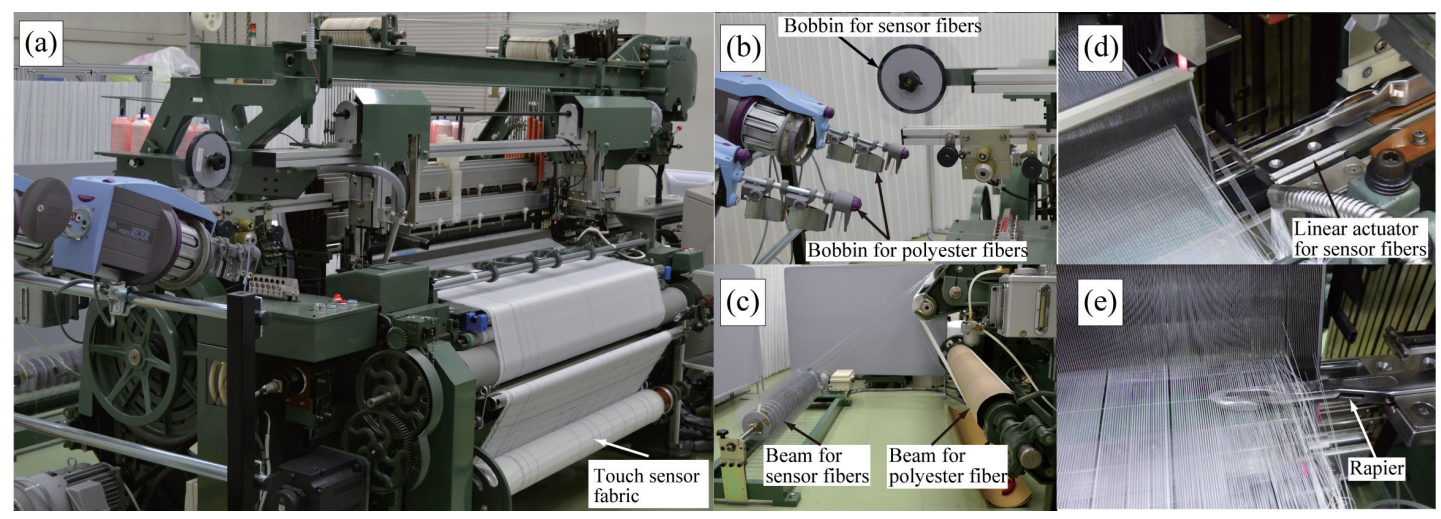

Fig. 3. (Color online) Photographs of developed automatic looming machines. (a) Overall looming machine. (b) Bobbins for sensor and polyester fibers. (c) Beams for sensor and polyester fibers. (d) Linear actuator for sensor fiber weaving. (e) Conventional rapier for polyester fibers. 


\section{Evaluation of Weaving Sensor Fabric with Automatic Looming System}

\subsection{Density of touch sensors}

By using the developed automatic looming system, the sensor density of the touch sensor in the area of the fabric is tuned by adjusting the arrangement of the sensor weft and warp fibers in the fabric. The sensing points of our touch sensor fabric are the intersection points of the sensing weft and warp fibers. Thus, the pitch between sensor fibers including both sensor weft and warp fibers defines the sensor density in the sensor fabric. For example, the sensor density in a certain area is 1 sensor $/ \mathrm{cm}^{2}$ if the sensor pitch of the weft and warps is $1 \mathrm{fiber} / \mathrm{cm}$. Therefore, the sensor density of our fabric is controlled by changing the pitch between sensor fibers.

The different methods of changing the pitch of sensor wefts and warps are as follows. To arrange sensor warps, the deployment of sensor fibers in warps is defined at the reed of the automatic looming machines as shown in Fig. 4(a). Basically, the longitudinal warp fibers are thrown from the back beam through the heddle and reed to the takeup roll. The weaving machine defines the sensor fiber arrangement at the beam and interlaces the weft fibers to fix the arranged warps and form the sensor fabric. The reed has teeth at certain gaps, and the sensor fibers are thrown between teeth at the desired points of the reed. The gap between teeth of the reed, number of teeth, and pitch of the sensor fibers are $G_{\text {warp }}, n$, and $P_{\text {warp }}$, respectively. The pitch of the sensor fibers $P_{\text {warp }}$ is determined.

$$
P_{\text {warp }}=G_{\text {warp }} \times n
$$

By changing $n$ and $G_{\text {warp }}$, the sensor fibers with several different pitches are woven as shown in Fig. 4(b). The different pitches are $5 \mathrm{~mm}, 10 \mathrm{~mm}, 5 \mathrm{~cm}, 10 \mathrm{~cm}, 50 \mathrm{~cm}$, and $1 \mathrm{~m}$ from the leftmost sensor fibers, and their corresponding numbers of teeth between sensor fibers are $5,10,50,100,500$, and 1000 when $G_{\text {warp }}$ is $1 \mathrm{~mm}$. In these ways, the

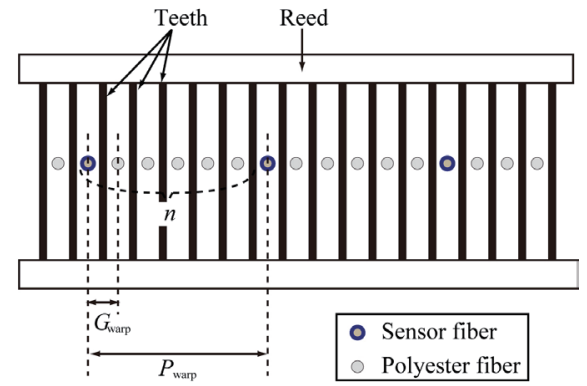

(a)

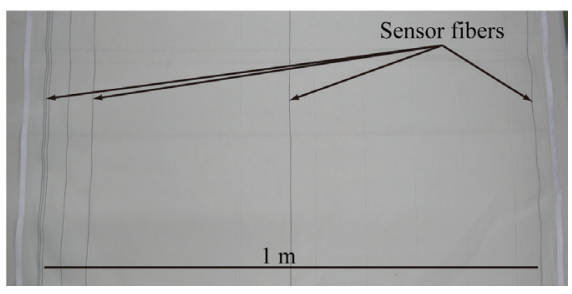

(b)

Fig. 4. (Color online) (a) Schematic views of reed. The reed has teeth with certain pitches, and the sensor fibers pass certain pitches and are thrown between teeth at the desired points of the reed. (b) Sensor warps with different pitches of $5 \mathrm{~mm}, 10 \mathrm{~mm}, 5 \mathrm{~cm}, 10 \mathrm{~cm}, 50 \mathrm{~cm}$, and $1 \mathrm{~m}$. 
pitch of the weft sensor fibers can be decreased to $5 \mathrm{~mm}$, thus our weaving machine can offer sufficient sensor density on the wefts (i.e., several centimeters) because the average width of a human foot heel is $6.3 \mathrm{~cm}$. Note that by using a narrower fiber and a smaller gap between teeth of the reed, a lower sensor density will be possible.

To arrange sensor wefts, the number of polyester fiber wefts between sensor fibers is changed using a dobby machine of an automatic weaving system when the sensor fibers and polyester fibers are interlaced between warps. The following three steps are repeated for weaving. 1) The warps are wound off and rolled up on a take-up roll at a certain length. 2) One part of the warp moves upwards and the other moves downwards. 3) The wefts are interlaced between upper and lower warps. The certain moving length for one weft is a unit for changing the pitch of the sensor wefts. The number of polyester wefts between sensor fibers defines the pitch of the sensor fibers as shown in eq. (2):

$$
P_{\text {weft }}=L_{\text {polyester-weft }} \times n+L_{\text {sensor-weft }} \text {, }
$$

where certain moving lengths of the polyester fiber and sensor fiber, number of polyester fibers, and pitch of the sensor weft fibers are $L_{\text {polyester-weft, }}, L_{\text {sensor-weft }}, n$, and $P_{\text {weft }}$, respectively. The number of polyester fibers is controlled using a dobby machine in our weaving machine. By changing the number of polyester fibers between sensor fibers, the pitch of sensor wefts is changed from $5 \mathrm{~mm}$ to $1 \mathrm{~m}$, as shown in Fig. 5. The different pitches of sensor wefts are $5 \mathrm{~mm}, 10 \mathrm{~mm}, 5 \mathrm{~cm}, 10 \mathrm{~cm}, 50 \mathrm{~cm}$, and $1 \mathrm{~m}$ from the lowermost of the sensor fibers. Their corresponding numbers of polyester fibers between sensor fibers are $6,14,77,156,786$, and 1573 when the moving length per one polyester weft is $0.635 \mathrm{~mm}$ and the moving length per sensor fiber is defined as $1 \mathrm{~mm}$. Thus, the pitch of the sensor weft can be tuned for sensors detecting human hands or feet. ${ }^{(5)}$

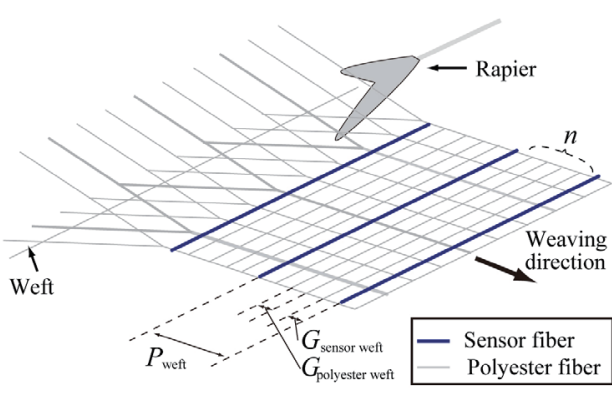

(a)

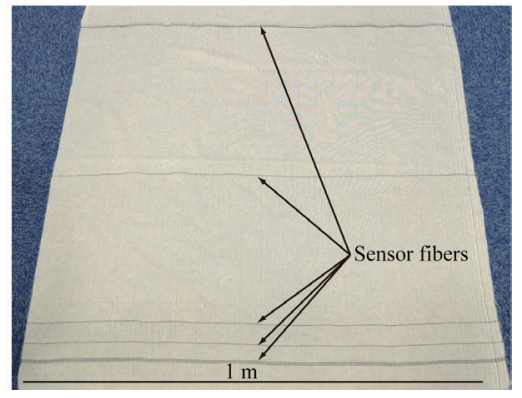

(b)

Fig. 5. (Color online) (a) Schematic views of sensor weft weaving. The pitch of the sensor wefts is tuned by changing the number of polyester fibers between sensor fibers. (b) Sensor weft with different pitches of $5 \mathrm{~mm}, 10 \mathrm{~mm}, 5 \mathrm{~cm}, 10 \mathrm{~cm}, 50 \mathrm{~cm}$, and $1 \mathrm{~m}$. 
Finally, Fig. 6 shows the sensor fabric that has different pitches for both wefts and warps of sensor fibers. The pitches of wefts and warps are the same as above (i.e., $5 \mathrm{~mm}$, $10 \mathrm{~mm}, 5 \mathrm{~cm}, 10 \mathrm{~cm}, 50 \mathrm{~cm}$, and $1 \mathrm{~m}$ ). Therefore, by changing the pitches of wefts and warps, the sensor density (i.e., several sensors per square centimeter) can be tuned for human position or walk monitoring. ${ }^{(5)}$

\subsection{Capacitive sensitivity control of touch sensors by weaving}

The capacitive sensitivity of our touch sensor fabric is controlled by changing the coverage of the polyester fibers on sensor fibers. In principle, the sensitivity of a capacitive touch sensor is defined by the thickness of dielectric layers on the sensing electrode. ${ }^{(23)}$ The dielectric layer of our fabric touch sensors is not only the UV-curable polymer on the conductive-polymer electrode but also the polyester fibers on the sensor fibers, while conventional touch sensors have only the dielectric film on the electrode. Thus, the coverage of the polyester fibers on the sensing conductive-polymer-coated sensing fibers changes the sensitivity of the touch sensors.

In this section, the coverage of sensor wefts with polyester fibers is controlled. Figure 7(a) shows that sensor wefts are interlaced in the open space between upper and lower warps for weaving. Basically, warps are passed from the back beam through
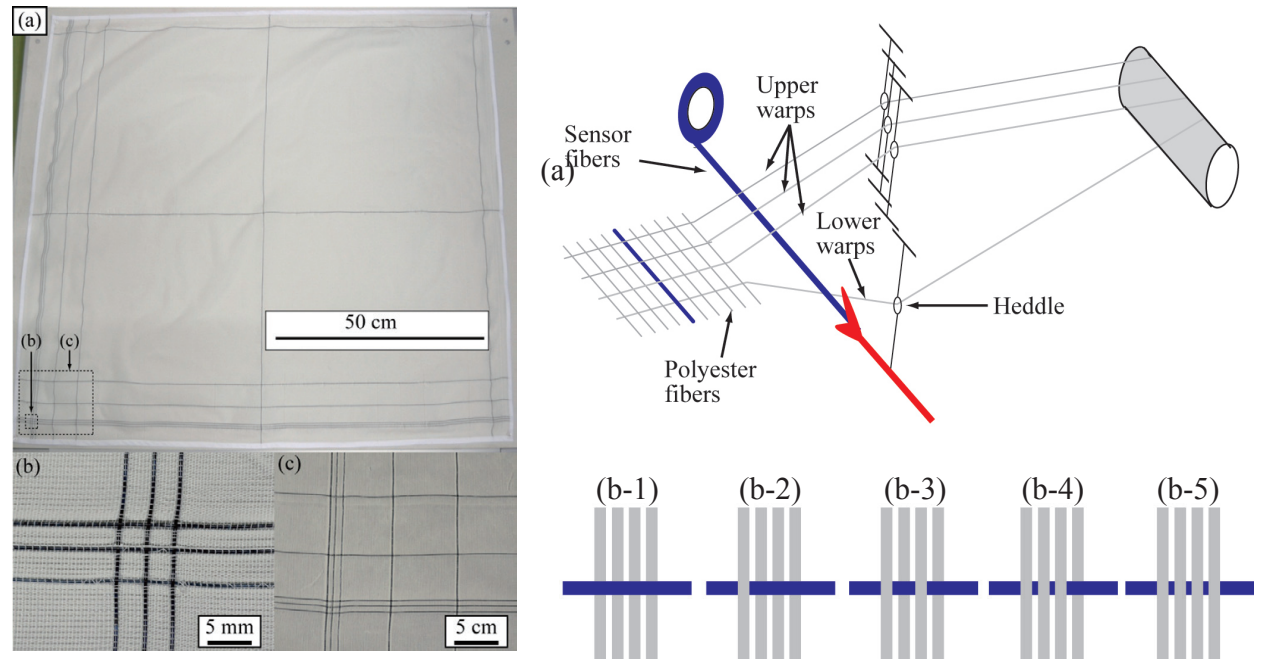

Fig. 6 (left). (Color online) (a) Photograph of woven sensor fabric that has sensor wefts and warps with different pitches of $5 \mathrm{~mm}, 10 \mathrm{~mm}, 5 \mathrm{~cm}, 10 \mathrm{~cm}, 50 \mathrm{~cm}$, and $1 \mathrm{~m}$. (b) Magnified photograph of sensor wefts and warps with $5 \mathrm{~mm}$ pitch. (c) Magnified photograph of sensor fibers with $5 \mathrm{~mm}, 10$ $\mathrm{mm}, 5 \mathrm{~cm}$, and $10 \mathrm{~cm}$ pitches.

Fig. 7 (right). (Color online) (a) Schematic view of control of polyester coverage on sensor fibers. The ratio between upper and lower warps is changed to increase or decrease the coverage ratio of polyester fibers on the sensing fibers. (b) Different coverage ratios of polyester fibers on sensing fibers. 
the heddles and reed to the front take-up roll. Heddles are fixed on the four heddle frames (the four frames are conventional, and more frames are utilized depending on the applications). The warps are opened for weft interlacing by moving the heddle frame up or down. The ratio between the upper and lower warps is changed by using the dobby machine of our automatic looming machine in order to increase or decrease the coverage ratio of polyester fibers on the sensing fibers, as shown in Fig. 7(a). In Fig. 7(a), the three left heddle frames are above the sensor weft, and the right one is below the weft; thus, $75 \%$ of the polyester fibers are on the sensor weft. Figure 7(b) shows a different coverage ratio. In the case of (b-1), no polyester fiber covers the sensor fiber, which is $0 \%$ coverage. The sensor fiber is placed on the fabric without fixing the fiber on the fabric and the capacitance change under pressure is measured in the same way as for the other conditions. In the case of (b-2), one of four polyester fibers covers the sensor fibers showing a coverage of $25 \%$. In the same way, the coverages of (b-3), (b-4), and (b-5) are 50,75 , and $100 \%$, respectively. Note that the sensor wefts in the cases of (b-1) and (b5) cannot be fixed with wefts, and the fabric with the coverage between $25-75 \%$ can be woven with automatic looming machines. The coverages of 0 and $100 \%$ are required for additional sewing or stitching for fixing the sensor fiber of polyster fibers on the sensor fabric. Figure 8 shows the sensor fabrics with different coverages of polyester fibers on sensor fibers. The ratio of the polyester fibers on sensor fibers is changed by changing the weaving.

The capacitive sensitivity of our woven fabric is evaluated with the experimental setup shown in Fig. 9(a). The sensors are first connected to a conventional capacitance

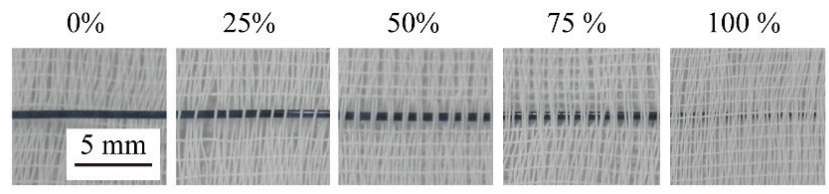

Fig. 8. (Color online) Photographs of different coverage ratios of polyester fibers on sensor fibers.

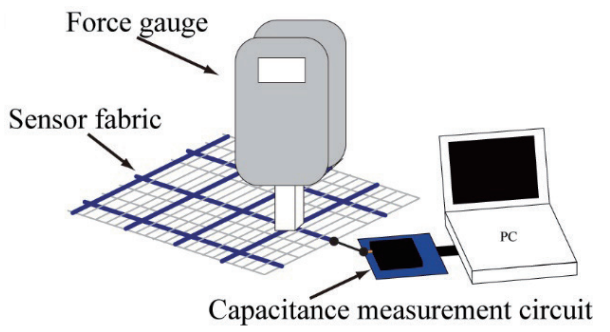

(a)

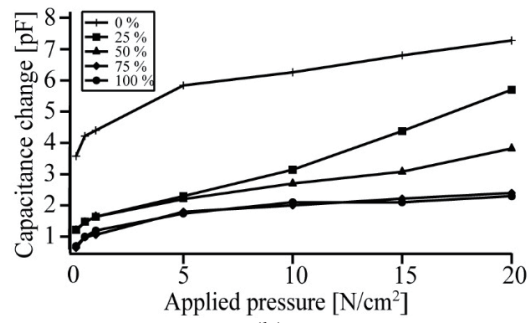

(b)

Fig. 9. (Color online) (a) Experimental setup of capacitive sensitivity of touch sensor fabric. (b) Relationship between applied pressure and capacitance changes of sensor fibers with different polyester fiber coverage ratios. 
measurement circuit in a microcontroller unit (MCU) of C8051F700 DK, manufactured by Silicon Laboratories Inc., and the ground line is connected to the aluminum electrode that serves as an alternative to the human body. The aluminum electrode is $2 \mathrm{~cm}$ wide, which is the average width of a human finger. The pressure applied to the sensor ranges from $0-20 \mathrm{~N} / \mathrm{cm}^{2}$. The several $\mathrm{N}$ is the average pressure of a human hand when touching the sensors. The applied pressure is tuned by a force gauge with an integrated z-axis automatic positioning stage (Aikoh Engineering, MODEL-FTN1-13A). Figure 9(b) shows the relationship between applied pressure and the resultant capacitance changes. The capacitance change increases when the applied pressure is increased. Even under a small pressure of $0.1 \mathrm{~N} / \mathrm{cm}^{2}$, a capacitance change of $2 \mathrm{pF}$ is measured. This means that human touch with small pressure can be detected with our sensor. On the other hand, in the case of $25 \%$ coverage of the sensor fibers, the capacitance exhibits its largest increase with pressure. This excludes the response of $0 \%$ coverage of sensor fibers that cannot be fabricated with the automatic looming machine. In case of $0 \%$ coverage of the sensor fiber, the human finger directly touched the sensor fibers as there were no polyester fibers, resulting in large capacitance change. In the other cases, the polyester fibers come in between the sensor fibers and human fingers forming textile structures and they reduce the contact area between the sensor fibers and the human finger. The response is almost double that of the $75 \%$ coverage sensor fibers. Therefore, the smallest coverage of the sensor fiber (i.e., 25\%) is required for high-capacitance sensitivity of the fabric touch sensor. Thus, this coverage of sensor fibers is utilized for actual weaving of large sensor fabrics.

\subsection{Capacitive sensitivity of meter-scale long sensor fabric}

Figure 10(a) shows that pressure is applied at different contact points of the sensor fibers. To change the contact points, the contact points of the sensor fiber are $0,10,20$, $30,40,50,60,70,80,90$, and $100 \mathrm{~cm}$ far from the capacitance measurement circuits. Figure 10(b) shows the relationship between the distance between contact points and the

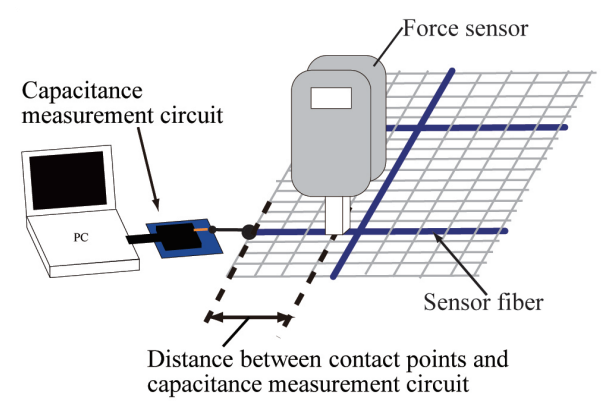

(a)

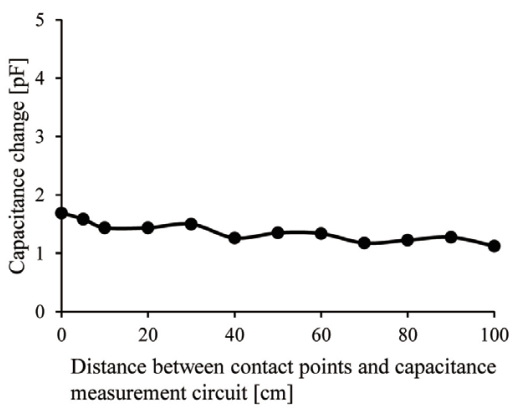

(b)

Fig. 10. (Color online) (a) Capacitance change at different points of the sensor fabric is measured. The distance between the contact points and the capacitance measurement circuit is changed for changing the contact points. (b) Relationship between distance between the contact points and the capacitance measurement circuit and capacitance changes. 


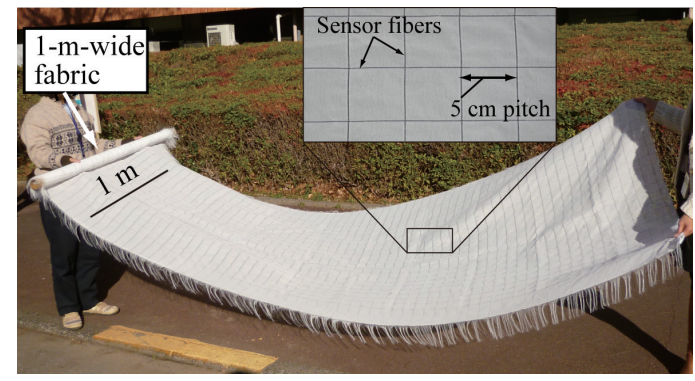

Fig. 11. (Color online) Demonstration of weaving of roll of sensor fabric with an area of $1.2 \times 5$ $\mathrm{m}^{2}$.

capacitance measurement circuit and the capacitance changes when the applied pressure and the size of the counter aluminum electrodes are $4.9 \mathrm{~N}$ and $2 \times 2 \mathrm{~cm}^{2}$. The resultant capacitance changes decrease when the contact points are far from the capacitance measurement circuit. This is because the conductive polymer has high resistivity and the output signal is decreased through the high resistive long sensor fibers. However, the output capacitance at the distance of $100 \mathrm{~cm}$ is over $1 \mathrm{pF}$, which is sufficient for measurement with a conventional capacitance measurement circuit.

\section{Demonstration of Automatic Weaving of Meter-Scale Touch Sensor Fabric}

Finally, we demonstrated the weaving of a $1.2 \times 5 \mathrm{~m}^{2}$ meter-scale touch sensor fabric with our automatic looming machine (Fig. 11). The photo in Fig. 11 shows the roll of the sensor fabric carried by two humans. Also, $1.2 \mathrm{~m}$ is the standard width of the fabric woven by automatic looming machines, and this is sufficiently wide to manufacture carpet-type and bed-sheet-type touch sensor systems. In addition, the sensitivity area of the sensor fibers is 400 sensors $/ 1 \mathrm{~m}^{2}$ where the sensor weft and warp fibers are arranged at a $5 \mathrm{~cm}$ pitch for detecting human feet (average width of human foot heel: $6.3 \mathrm{~cm}$ ). The sensor coverage of the polyester fiber on the wefts is $25 \%$, which is produced by the method described in $\S 3.2$. Therefore, our woven sensors are suited for human monitoring in nursing homes and hospitals.

\section{Conclusions}

We have proposed and developed a 1.2-m-wide automatic looming machine to continuously construct a meter-scale floor touch sensor fabric. To develop our automatic looming system, a conventional automatic looming machine was modified to weave the thick and sensitive sensing fibers as wefts and warps at the desired pitches. The looming machine has a new rapier of the linear actuator for sensing wefts, which carries the wefts in the middle of the gap between the upper and lower warps in order to avoid any friction between warps and sensing fibers. Two separated back beams for sensor fibers and polyester fibers are equipped for controlling the different tensions of the two fibers. 
By using our automatic looming machine, weaving of a $1.2 \times 5 \mathrm{~m}^{2}$ fabric with a 5 $\mathrm{cm}$ sensor fiber pitch is demonstrated. The pitch of the sensor weft and warp fibers is then shortened to $5 \mathrm{~mm}$ using a dobby machine and a reed of the looming machine. In addition, the capacitive sensitivity to touch input is tuned by controlling the coverage of sensor fibers with dielectric conventional polyester fibers. The output capacitance change at the smallest coverage of $25 \%$ is approximately twofold that at the coverages of 75 and $100 \%$. These weaving sensor fabrication techniques will provide a system for manufacturing meter-scale floor and bed touch sensors in nursing homes and hospitals.

\section{Acknowledgements}

This research was conducted as an undertaking of the New Energy and Industrial Technology Development Organization (NEDO) project entitled "Development of Manufacturing Technologies for Hetero-Functional Integrated Devices” (BEANS project).

\section{References}

1 F. Viani, F. Robol, A. Polo, P. Rocca, G. Oliveri and A. Massa: Proc. IEEE 101 (2013) 2381.

2 R. Fukui, T. Mori and T. Sato: Adv. Robo. 26 (2012) 1127.

3 D. Ding, R. A. Cooper, P. F. Pasquina and L. Fici-Pasquina: Maturitas 69 (2011) 131.

4 Y. Visell, A. Law and J. Cooperstock: IEEE Trans. Haptic. 2 (2009) 148.

5 Digital Human Consortium Japan (2004): Human Body Properties Database [Online]. http:// www.dh.aist.go.jp/bodyDB/index-e.html (accessed on January 2014).

6 A. Sixsmith and N. Johnson: IEEE Pervasive Comput. 3 (2004) 42.

7 W. Fang, E. Stone, M. Skubic, J. M. Keller, C. Abbott and M. Rantz: IEEE J. Biomed. Health Inf. 17 (2013) 346.

8 H. Rimminen, J. Lindström, M. Linnavuo and R. Sepponen: IEEE Trans. on Inf. Technol. Biomed. 14 (2010) 1475.

9 T. Nakano, E. Koyama, T. Nakamura, T. Ito, K. Tamura and M. Yaginuma: Psychiatry Clin. Nurosci. 56 (2002) 287.

10 P. Zappi, E. Farella and L. Benini: IEEE Sens. J. 10 (2010) 1486.

11 M. Narducci, L. Yu-Chia, W. Fang and J. Tsai: J. Micromech. Microeng. 23 (2013) 055007.

12 M. Esashi: Jpn. J. Appl. Phys. 51 (2012) 080001.

13 J. Lu, H. Takagi, Y. Nakano and R. Maeda: Microsyst. Technol. 19 (2013) 775.

14 D. Taroata, W. Fischer, T. A. Cheema, G. Garnweitner and S. Gunter: IEEE Trans. Dielectr. Electr. Insul. 19 (2012) 298.

15 G. Mattana, T. Kinkeldei, D. Leuenberger, C. Ataman, J. Ruan, F. Molina-Lopez, A. Vasquez Quintero, G. Nisato, G. Troster, D. Briand and N. de Rooij: IEEE Sens. J. 13 (2013) 3901.

16 C. Muller, M. Hamedi, R. Karlsson, R. Jansson, R. Marcilla, M. Hedhammar and O. Inganas: Adv. Mater. 23 (2011) 898.

17 E. Post, M. Orth, P. Russo and N. Gershenfeld: IBM Syst. J 30 (2000) 840.

18 G. Kita, M. Shikida, Y. Suzuki, Y. Tsuji and K. Sato: Micro Nano Lett. 5 (2010) 389.

19 G. Kita, M. Shikida, Y. Suzuki and K. Sato: Micro Nano Lett. 5 (2010) 210.

20 Y. Hasegawa, M. Shikida, D. Ogura and K. Sato: J. Micromech. Microeng. 18 (2008) 085014.

21 S. Takamatsu, T. Kobayashi, N. Shibayama, K. Miyake and T. Itoh: Sens. Actuators, A 184 (2012) 184.

22 S. Takamatsu, T. Yamashita, T. Imai and T. Itoh: Sens. Mater. 25 (2013) 627.

23 G. Barrett and R. Omote: Inf. Disp. 3 (2010) 16. 